\title{
Comunidade de Euglossini (Hymenoptera, Apidae) das dunas litorâneas do Abaeté, Salvador, Bahia, Brasil
}

\author{
Blandina Felipe Viana ${ }^{1,2}$ \\ Astrid Matos Peixoto Kleinert ${ }^{3}$ \\ Edinaldo Luz das Neves ${ }^{1}$
}

\begin{abstract}
Community of Euglossini (Hymenoptera, Apidae) from the coastal sand dunes of Abaeté, Salvador, Bahia, Brazil. The Euglossini community structure was analyzed by attracting males with the scents eucalyptol, eugenol, vanillin, benzyl benzoate and methyl salicylate, and by netting bees on flowers. The samplings took place three times a month along one year from 6:00 a.m. to 6:00 p.m. The scent baits attracted 670 individuals belonging to seven species of three genus. The predominant species were Euglossa cordata (Linnaeus, 1758) (76.6\%) and Eulaema nigrita Lepeletier, 1841 (21.8\%). Euglossini males visited the scents along the whole year, being more abundant in May and in August. The most efficient fragrance was eucalyptol, attracting 624 individuals of five species. The males abundance fluctuated along the day, being the highest frequency observed between 8:00 a.m. to 10:00 a.m. Forty eigth Euglossini females of four species were netted visiting flowers of 14 plant species belonging to 13 families. Solanaceae and Caesalpiniaceae were the most visited. The species catched on flowers were Euglossa cordata, Eulaema nigrita, Euplusia mussitans (Fabricius, 1787) and Eulaema meriana flavescens Friese 1899. Euglossa cordata was the predominant species on flowers (64.6\%), being collected during almost the whole year. Euplusia mussitans was the only species netted on flowers which males were not sampled on the scents.
\end{abstract}

KeYwORDs. Community structure; Euglossini; richness; scent baits; sand dunes.

\section{INTRODUÇÃO}

Os Euglossini são abelhas com distribuição, praticamente, restrita à Região Neotropical. Os limites ao norte são as regiões de Bownsville, Texas e Silverbell, Arizona (MINCKLEY \& REYES 1996). Ao sul, são encontrados representantes deste grupo em Cordoba, Argentina (Moure 1967) e nas florestas do Rio Grande do Sul (Wittmann et al. 1988).

Sua maior diversidade encontra-se nas zonas quentes e úmidas equatoriais (Moure 1967), sendo consideradas importantes vetores de pólen de muitas espécies botânicas, principalmente da família Orchidaceae (DRESSLER 1982; RouBIK 1989). As fêmeas coletam pólen e néctar nas flores de algumas espécies vegetais (HeIthaus 1979; CAMARgo \& MazUCATO 1984). Os machos, além de visitarem as flores para coletar néctar, também o fazem em busca de substâncias aromáticas produzidas por certas plantas, como aquelas da família Orchidaceae, e por fontes extra-florais (Dodson 1966; DrESSLER 1982; Williams 1982; Ackerman 1983a). Estes compostos servem provavelmente para atrair outros machos co-específicos e formar uma agregação: comportamento necessário para que as fêmeas sejam atraídas e ocorra o acasalamento (PERUQueTtI 2000).

A utilização de compostos sintéticos, análogos àqueles produzidos nas flores, como iscas para atração de machos, é uma importante ferramenta que permite a obtenção de dados mais completos sobre a ecologia de comunidades desse grupo de abelhas (Pearson \& Dressler 1985; RoubiK \& Ackerman 1987; Oliveira \& CAMPOS 1995; RebÊLo \& GaróFalo 1997), já que apenas coletas nas flores não fornecem dados suficientes sobre a diversidade desse grupo em uma dada localidade (Rebêlo \& Garófalo 1991; Neves \& Viana 1997; Peruquetti et al. 1999; BEZERRA \& MARTINS 2001).

Através de coletas periódicas nas flores e em iscas-odores, a fauna de Euglossini das dunas litorâneas do Abaeté foi estudada, visando obter informações sobre alguns aspectos ecológicos do grupo, tais como: riqueza em espécies, abundância relativa, flutuação sazonal, horário de atividade,

1. Laboratório de Biologia e Ecologia de Abelhas (LABEA), Departamento de Zoologia, Instituto de Biologia, Universidade Federal da Bahia. Rua Barão de Geremoabo s/n, Campus Universitário de Ondina, 40170-110 Salvador-BA, Brasil. Endereço eletrônico: blandefv@ufba.br

2. Programa de Pós-Graduação em Ecologia e Biomonitoramento, Universidade Federal da Bahia. Endereço eletrônico: ecologia@ufba.br

3. Departamento de Ecologia, Instituto de Biociências, Universidade de São Paulo. Rua do Matão, Travessa 1, n 327, 05508-900 São Paulo-SP, Brasil. Endereço eletrônico: astridk1@usp.br 
preferência por compostos aromáticos e recursos florais utilizados.

\section{MATERIAL E MÉTODOS}

O estudo foi desenvolvido em uma área de dunas costeiras coberta com vegetação de restinga na Área de Proteção Ambiental das Lagoas e Dunas de Abaeté (12²56'S e 38²1'W), Salvador, Bahia no período de fevereiro de 1996 a janeiro de 1997. As coletas foram realizadas três vezes ao mês, das 6:00 às 18:00 h, totalizando $432 \mathrm{~h}$ de amostragem.

Para a captura dos machos foram selecionados os compostos eucaliptol, eugenol, salicilato de metila, benzoato de benzila e essência de baunilha, colocados individualmente em armadilhas e em chumaços de algodão enrolados em gaze, conforme método descrito por Neves \& Viana (1999), a fim de serem utilizados como iscas-odores.

Armadilhas e chumaços foram pendurados nos ramos de arbustos a de 1,7 $\mathrm{m}$ de altura, distribuídos na área a uma distância de aproximadamente $10 \mathrm{~m}$ uns dos outros. As armadilhas foram vistoriadas a cada $60 \mathrm{~min}$, quando eram reabastecidas com essências e os indivíduos, ali aprisionados, retirados. Os machos atraídos aos chumaços, eram capturados diretamente com rede entomológica.

Os visitantes florais foram amostrados no mesmo local, entre janeiro e dezembro de 1996, em uma área de 8,2 ha. A captura das abelhas nas flores foi feita pelo método de varredura, utilizando-se rede entomológica, de acordo com os procedimentos descritos por SAKAGAMI et al. (1967). O tempo de espera em cada planta florida foi de, no máximo, 10 minutos.

Nos dias de coleta foram anotados os dados de temperatura e umidade relativa do ar, utilizando-se um termohigrômetro colocado no meio da área amostrada a uma altura de aproximadamente $1,5 \mathrm{~m}$ do solo. Os dados de precipitação mensal foram obtidos na estação meteorológica do Aeroporto Deputado Luís Eduardo Magalhães, Salvador, BA.

Para a determinação da estrutura etária da população de machos, o padrão de desgaste alar foi avaliado segundo a classificação proposta por RÊBELO \& GARÓFALO (1991): idade 1: sem desgaste alar; idade 2: pequeno desgaste alar; idade 3: moderado desgaste alar e idade 4: grande desgaste alar.

Para verificar se houve variação significativa anual e diária na abundância relativa dos indivíduos foi aplicado o teste do $\chi^{2}$.

\section{RESULTADOS}

Visitando as essências, foram coletados 670 machos pertencentes a três gêneros (Tabela I). Euglossa cordata foi a espécie mais abundante, representando 76,6\% da amostra.

A essência mais atrativa foi o eucaliptol, com $93 \%$ do total das capturas, seguida por benzoato de benzila (3\%) e salicilato de metila (2,6\%); as menos visitadas foram o eugenol e a baunilha, tendo cada uma atraído apenas $0,7 \%$ do total dos visitantes. Quase todos os machos de Euglossa cordata e
Eulaema nigrita foram atraídos por eucaliptol, enquanto que metade dos machos coletados de Eulaema meriana flavescens e todos os indivíduos de Eulaema bombiformis niveofasciata foram atraídos pelo salicilato de metila. O único indivíduo de Eulaema cingulata capturado foi atraído por eugenol.

Embora os machos tenham visitado os compostos durante todo o ano de estudo, isso não ocorreu de forma homogênea, havendo variação significativa no número de indivíduos entre os meses de coleta $\left(\chi^{2}=136,69 ;\right.$ g.l. $\left.=11 ; \mathrm{p}<0,001\right)$. As maiores freqüências de indivíduos foram observadas durante o período mais chuvoso (abril a agosto), com o máximo de abundância no mês de maio (Fig. 1).

Com relação ao horário de atividade, os machos de Euglossini apresentaram uma variação significativa no número de indivíduos coletados $\left(\chi^{2}=28,75\right.$; g.l. $\left.=5 ; \mathrm{p}<0,001\right)$, ocorrendo uma maior freqüência entre 8:00 e 10:00 horas (Tabela II).

Quanto à estrutura etária das populações de machos, os indivíduos de idade 2 predominaram durante todo o ano. Machos das idades 1 e 3 também foram amostrados durante todo o ano, mas com menor predominância. Machos de idade 4 foram os menos freqüentes e estiveram ausentes nos meses de março e outubro (Fig. 2).

Em visita ao eucaliptol, três machos de Eulaema nigrita e dois de Euglossa cordata foram coletados transportando polínias. Os machos de Eulaema nigrita e um de Euglossa cordata portavam polínias de Catasetum sp. (Orchidaceae). Não foi possível identificar a que espécie de planta pertencia a polínia transportada pelo outro indivíduo de Euglossa cordata. Os exemplares de Eulaema com polínia de Catasetum $\mathbf{s p}$. foram coletados em janeiro, julho e dezembro e o de Euglossa, em novembro; o outro indivíduo de Euglossa, com polínia desconhecida, em dezembro.

Dentre os visitantes florais, foram coletadas 48 fêmeas de Euglossini pertencentes a quatro espécies, sendo Euglossa cordata a espécie predominante, com $64,6 \%$ do total dos indivíduos amostrados. Euplusia mussitans foi a única espécie coletada nas flores que não teve machos amostrados nas essências (Tabela III).

As abelhas foram coletadas em 14 espécies de plantas pertencentes a 13 famílias. Solanaceae e Caesalpiniaceae foram as famílias mais visitadas (Tabela IV).

Euglossa cordata, espécie mais abundante, foi amostrada durante todo o ano, exceto nos meses de janeiro e novembro de 1996. A única representante de Eulaema meriana flavescens foi coletada no mês de dezembro (Fig. 3).

\section{DISCUSSÃO}

Diversos estudos sobre comunidades de Euglossini empregando compostos aromáticos para a atração de machos, têm sido realizados em muitas localidades nos neotrópicos (JANZen et al. 1982; Ackerman 1983a; PeArson \& Dressler 1985; RoubiK \& ACKERMAN 1987; RebÊLo \& GARÓFALO 1991).

Embora as comparações entre os trabalhos, principalmente em relação à riqueza e abundância, devam ser feitas de forma 
Tabela I. Número de machos de Euglossini, atraídos por essências, em Abaeté, Salvador, Bahia, no período de fevereiro de 1996 a janeiro de 1997.

\begin{tabular}{lcc}
\hline Espécies de Euglossini & \multicolumn{2}{c}{ Sub-Total } \\
\hline & $\mathrm{N}$ & $\%$ \\
Euglossa cordata (Linnaeus, 1758) & 509 & 76,0 \\
Eulaema nigrita Lepeletier, 1841 & 150 & 22,5 \\
Eulaema meriana flavescens (Friese, 1899) & 6 & 0,9 \\
Eulaema bombiformis niveofasciata & & \\
(Friese, 1899) & 2 & 0,3 \\
Euglossa liopoda Dressler, 1982 & 1 & 0,1 \\
Eulaema cingulata (Fabricius, 1804) & 1 & 0,1 \\
Exaerete smaragdina (Guérin, 1845) & 1 & 0,1 \\
\hline Total de machos & 670 & 100 \\
\hline
\end{tabular}

cautelosa devido à falta de padronização dos métodos (MORATO 1998), o número de espécies encontrado neste estudo pode ser considerado representativo da comunidade local de Euglossini, quando comparado à fauna desse grupo de abelhas amostrada em pesquisas realizadas em alguns ecossistemas da Região Nordeste, Sul e Sudeste do Brasil (Raw 1989; WittMann et al. 1988; Rebêlo \& Garófalo 1991; Peruquetti et al. 1999; Neves \& Viana 1997, 1999; Jesus 2000). Porém, pode ser considerada pobre quando comparada aos levantamentos realizados nas florestas úmidas da América Central e Região Amazônica (Pearson \& Dressler 1985; Roubik \& Ackerman 1987; Morato \& Campos 1992; Oliveira \& Campos 1995; Silva \& Rebêlo 1999; REBÊlo \& SiLVA 1999), onde o número de espécies amostradas foi, na maioria dos casos, bem maior do que o obtido neste trabalho.

Quanto à abundância, a predominância de duas espécies dentre as sete amostradas, pode estar representando o padrão da estrutura da comunidade local ou refletindo a fraca associação das espécies pouco representadas com as essências utilizadas.

Vários autores têm verificado que, apesar de utilizarem muitos compostos, o padrão de preferência tem se repetido. Os compostos utilizados nesse estudo estão entre aqueles destacados por Williams \& WhitTen (1983) como os mais eficientes na atração de machos.

Dentre as fragrâncias utilizadas, o eucaliptol foi a mais atrativa, tanto em número de indivíduos, como em número de espécies. Esses dados são semelhantes aos obtidos por outros estudos que também empregaram o eucaliptol (RAw 1989; Aguilar 1990; Neves \& Viana 1997, 1999; Bezerra \& Martins 2001).

Embora haja variação geográfica e/ou sazonal (WITTMANN et al. 1987; ACKERMAN 1989; RebÊLO \& GaróFAlo 1991; Oliveira \& CAMPOs 1996) na preferência por compostos por parte dos machos de uma mesma espécie - o que pode estar refletindo as variações nas disponibilidades de fontes naturais de recursos - na maioria dos estudos tem-se observado que um grande
Tabela II. Abundância relativa de machos de Euglossini atraídos por essências e médias de temperaturas e umidades relativas nos diferentes intervalos de horas, em Abaeté, Salvador, Bahia, no período de fevereiro de 1996 a janeiro de 1997; dp= desvio padrão.

\begin{tabular}{cccccc}
\hline \multirow{2}{*}{ Horário } & \multirow{2}{*}{ Temperatura $\left({ }^{\circ} \mathrm{C}\right)$} & \multicolumn{3}{c}{ Umidade Relativa $(\%)$} \\
\cline { 3 - 6 } & indivíduo & Média & $\mathrm{dp}$ & Média & $\mathrm{dp}$ \\
\hline 6:00-8:00 & 15,9 & 25,8 & 2,10 & 82,2 & 4,70 \\
8:00-10:00 & 35,9 & 28,6 & 2,30 & 74,4 & 5,20 \\
10:00-12:00 & 24,2 & 30,2 & 2,30 & 70,8 & 3,98 \\
12:00-14:00 & 12,5 & 30,4 & 2,47 & 69,6 & 3,38 \\
14:00-16:00 & 10,3 & 30,0 & 2,65 & 71,4 & 3,63 \\
16:00-18:00 & 1,2 & 27,9 & 2,76 & 78,0 & 4,62 \\
\hline
\end{tabular}

número de indivíduos de espécies como Euglossa cordata e Eulaema nigrita é coletado em eucaliptol em diferentes épocas do ano e em diferentes localidades (RAw 1989; AguILAR 1990; Rebêlo \& Garófalo 1991; Neves \& Viana 1997, 1999; BezerRa \& MARTINS 2001).

Apesar da constatada eficiência do uso de algumas essências na avaliação das comunidades locais de Euglossini, muitos autores têm observado que nem todas as espécies presentes em uma área são atraídas às iscas-odores (PEARSON \& Dressler 1985; RoubiK \& Ackerman 1987; Rebêlo \& Garófalo 1991; Neves \& Viana 1997; Jesus 2000; Bezerra \& MarTins 2001). Neste estudo, esse fato também ocorreu, pois fêmeas de Euplusia mussitans, cujos machos não foram amostrados nas iscas-odores, foram coletadas em ninhosarmadilha (VIANA et al. 2001a,b) e nas flores.

A flutuação sazonal dos indivíduos em Abaeté parece estar sendo influenciada, dentre outros fatores, pela precipitação, pois a maior freqüência de indivíduos nas iscas ocorreu no inicio da estação mais chuvosa (maio) e a menor, no final da estação menos chuvosa (março). Resultados similares foram encontrados por Rebêlo \& Garófalo (1991), em florestas semidecíduas, Peruquetti et al. (1999) em fragmentos de Mata Atlântica, na Região Sudeste, e OLIVEIRA (1999), em florestas de terra firme na Amazônia central, dentre outros.

A precipitação pode estar regulando a disponibilidade local

Tabela III. Número de fêmeas de Euglossini coletadas nas flores nas dunas de Abaeté, Salvador, Bahia, no período de janeiro a dezembro de 1996.

\begin{tabular}{lcc}
\hline Espécies de Euglossini & $\mathrm{N}^{\circ}$ de fêmeas & $\%$ \\
\hline Euglossa cordata (Linnaeus, 1758) & 31 & 64,6 \\
Eulaema nigrita Lepeletier, 1841 & 11 & 22,9 \\
Euplusia mussitans (Fabricius, 1787) & 5 & 10,4 \\
Eulaema meriana flavescens (Friese, 1899) & 1 & 2,1 \\
\hline Total de fêmeas & 48 & 100 \\
\hline
\end{tabular}


Tabela IV. Famílias e espécies botânicas visitadas por fêmeas de Euglossini nas dunas de Abaeté, Salvador, Bahia, entre janeiro e dezembro de 1996.

\begin{tabular}{|c|c|c|c|c|}
\hline Família & Espécie & $\begin{array}{c}\text { Recurso } \\
\text { oferecido }\end{array}$ & Espécie de Euglossini ( $\mathrm{n}^{\circ}$ de fêmeas) & $\begin{array}{l}\text { Total de } \\
\text { fêmeas }\end{array}$ \\
\hline Caesalpiniaceae & $\begin{array}{l}\text { Chamaecrista ramosa (Vog.) I \& B } \\
\text { var ramosa }\end{array}$ & Pólen & $\begin{array}{l}\text { Eulaema nigrita }(05), \text { Euglossa cordata }(03) \text {, } \\
\text { Euplusia mussitans }(03)\end{array}$ & 11 \\
\hline Solanaceae & Solanum stagnale Moric & Pólen & Eulaema nigrita (03), Euglossa cordata (08) & 11 \\
\hline Fabaceae & Centrosema brasilianum (L.) Benth & Néctar & Euglossa cordata (4), Euplusia mussitans (1) & 5 \\
\hline Lythraceae & Cuphea brachiata Koehne & Néctar & Euglossa cordata (3), Euplusia mussitans (1) & 4 \\
\hline Bignoniaceae & Tabebuia elliptica (DC) Sandw.. & Néctar & Euglossa cordata (03), Eulaema nigrita (01) & 4 \\
\hline Lamiaceae & Eriope blanchetti (Benth) R Harley & Néctar & $\begin{array}{l}\text { Eulaema nigrita (1), Eulaema meriana } \\
\text { flavescens (1), Euglossa cordata (1) }\end{array}$ & 3 \\
\hline Myrtaceae & Calycolpus legrandii Mattos & Néctar & Euglossa cordata (1) & 2 \\
\hline Clusiaceae & Kielmeyera argentea Choisy & Pólen & Euglossa cordata (1), Eulaema nigrita (1) & 2 \\
\hline Fabaceae & Acosmium bijugum (Vog.) Yakovl & Néctar & Euglossa cordata (1) & 1 \\
\hline Melastomataceae & Comolia ovolifolia DC Triana & Pólen & Euglossa cordata (1) & 1 \\
\hline Verbenaceae & Vitex cymosa Benth & Néctar & Euglossa cordata (1) & 1 \\
\hline Rubiaceae & Albertia $\mathbf{s p}$ & Néctar & Euglossa cordata (1) & 1 \\
\hline Ternstroemiaceae & Terstromia $\mathbf{s p}$ & Néctar & Euglossa cordata (1) & 1 \\
\hline Bromeliaceae & Aechmea $\mathbf{s p}$ & Néctar & Euglossa cordata (1) & 1 \\
\hline$\overline{\text { Total }}$ & & & & 48 \\
\hline
\end{tabular}

de recursos que, por sua vez, estaria alterando o padrão de nidificação e emergência das abelhas (ACKERMAN 1983a, b).

A migração inter-hábitat (JANZEN et al. 1982) é um outro fator que pode estar envolvido na flutuação sazonal de Euglossini, em Abaeté. Raw (1989), estudando o comportamento de dispersão entre áreas florestadas isoladas entre si, em Salvador, Bahia, pelo método de marcação e recaptura, obteve uma baixa taxa de recaptura, sugerindo através desse resultado que os machos não fixam residência em um local para o qual retornam regularmente e, desse modo, cobrem longas distâncias ao longo de sua vida.

A Área de Proteção Ambiental das Lagoas e Dunas de Abaeté é formada por um mosaico de ecossistemas como matas, dunas com vegetação de restinga e brejos. A flutuação populacional das abelhas no sítio escolhido pode estar sendo influenciada pelas populações residentes nos habitats vizinhos que viriam às dunas apenas para coletar recursos.

Embora tenham sido coletados ao longo de todo o dia, o horário de atividade dos euglossíneos, em Abaeté, foi maior no período da manhã, confirmando as observações de Dodson et al. (1969). Um período mais longo (9:00 às 16:00 horas) de atividade de vôo foi observado por Oliveira (1999), nas florestas da Amazônia central.
A temperatura do ar parece ser o principal controlador do horário de atividade dos Euglossini; por isso, essas abelhas tendem a evitar o forrageamento no período da tarde - quando a temperatura está mais alta - para evitar o superaquecimento do corpo (ARMBruster \& Berg 1994). Provavelmente, em Abaeté, as condições meteorológicas locais, entre 8:00 e 10:00 horas (temperatura e intensidade luminosa mais baixas e umidade mais alta), devem ter favorecido a atividade de forrageamento dessas abelhas.

Como o recurso oferecido manteve-se constante ao longo de todo o dia, sendo as armadilhas e chumaços abastecidos com essências a cada 60 minutos, esse padrão na atividade diária das abelhas pode estar refletindo também o período de oferta de substâncias aromáticas pelas plantas, demonstrando assim, a existência de estreitas relações entre abelhas e plantas secretoras de essências.

A predominância de machos jovens na amostra foi também observada por Zimmerman \& MAdrinan (1988) que, ao estudarem a estrutura etária de machos, verificaram que os mais jovens eram capturados coletando essências e os mais velhos, coletando néctar nas flores. A presença de maiores freqüências de indivíduos jovens nas essências foi confirmada por Rebêlo \& Garófalo (1991) e Neves \& Viana (1999), ao 


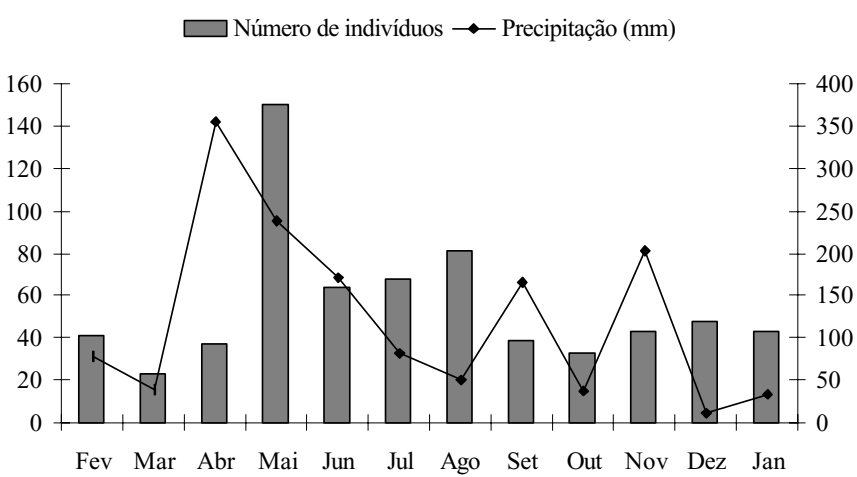

Fig. 1. Relação entre o número de machos de Euglossini atraídos às essências e a precipitação, nas dunas de Abaeté, Salvador, Bahia, no período de fevereiro de 1996 a janeiro de 1997.

analisarem a estrutura etária de espécimes atraídos por iscasodores. De acordo com ZimMERmAn \& MADRINAN (1988), este fato pode ser explicado pela necessidade que os machos mais jovens têm de coletar substâncias aromáticas antes de estabelecerem seus territórios, embora, ao longo de sua vida, possam abandonar seus territórios para novas coletas de fragrâncias.

A porcentagem de machos transportando polínias foi baixa $(0,7 \%)$ em relação ao total de indivíduos amostrados. Isso pode estar relacionado, provavelmente, ao pequeno número de indivíduos de orquídeas floridos dentro do raio de coleta dos euglossíneos, associado ao fato de que, além das orquídeas, essas abelhas coletam fragrâncias em outras fontes de odores.

Euglossíneos têm sido observados coletando resinas (ZuCCHI et al. 1969) e essências em plantas das famílias Leguminosae, Myrtaceae, Araceae, Gesneriaceae, Solanaceae, Euphorbiaceae e Bignoniaceae (DRESSLER 1967; DrESSLER 1982; Montalvo \& ACKERMAN 1986), em fungos (JANZEN et al. 1982), em frutas, folhas úmidas e troncos velhos (ACKERMAN 1983a) e em raízes expostas (WILLIAMs 1982).

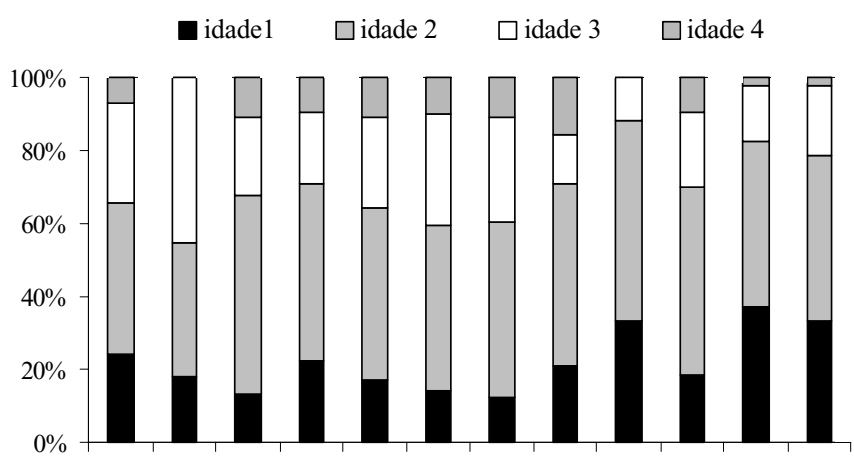

Fev Mar Abr Mai Jun Jul Ago Set Out Nov Dez Jan

Fig. 2. Abundância relativa, por idade alar, dos machos de Euglossini coletados nas essências, nas dunas de Abaeté, Salvador, Bahia, no período de fevereiro de 1996 a janeiro de 1997.



Fig. 3. Percentagem de fêmeas de Euglossini, coletados nas flores, nas dunas de Abaeté, Salvador, Bahia, no período de janeiro a dezembro de 1996.

Neste trabalho, a riqueza em espécies observada nas coletas nas flores corrobora o fato, já mencionado anteriormente, de que apenas coletas realizadas nas flores não são suficientes para a caracterização da diversidade local da fauna de Euglossini.

Apenas fêmeas de euglossíneos foram amostradas nas flores. Esse resultado está de acordo com a hipótese levantada por JANZEN et al. (1982), de que fêmeas e machos de uma mesma espécie exploram micro-hábitats diferentes. Entretanto, se contrapõe às observações feitas por CAMPOs et al. (1989), que revelam a ocorrência de machos e fêmeas de Euglossa towsendi Cockerell, 1904 coletando nas flores de um mesmo micro-hábitat em uma área de cerrado em Minas Gerais.

A predominância nas flores, assim como nas essências, de Euglossa cordata seguida de Eulaema nigrita, aliada ao fato de terem sido encontrados, na área de estudo, ninhos de Euglossa cordata em ramos de Agaristha revoluta (Spr.) D.C. (Ericaceae) e em ninhos-armadilha (VIANA et al. 2001a,b), sugerem que essas abelhas são residentes nessa área. Algumas espécies não predominantes também podem ser residentes na área como, por exemplo Euplusia mussitans, que foi amostrada em ninhos-armadilha (VIANA et al. 2001a,b) e nas flores.

Com relação às plantas visitadas, as fêmeas de Euglossini concentraram-se em espécies das famílias Caesalpiniaceae, Solanaceae, Fabaceae, Lythraceae, Bignoniaceae e Lamiaceae. Zucchi et al. (1969), Heithaus (1979) e Camargo \& Mazucato (1984) também destacaram as famílias Solanaceae, Bignoniaceae e Fabaceae como as mais visitadas por euglossíneos.

Chamaecrista ramosa (Vog.) I \& B var ramosa (Caesalpiniaceae), Cuphea brachiata Koehne (Lythraceae) e Eriope blanchetii (Benth) R Harley (Lamiaceae) estão, também, entre as espécies vegetais mais visitadas pela comunidade de Apoidea, em geral, em Abaeté (VIANA 1999), o que evidencia a não existência de uma relação, em nível específico, entre os euglossíneos e as plantas para a coleta de néctar e pólen. Embora estes tenham apresentado preferências florais, observa-se um comportamento relativamente polilético com relação à utilização dos recursos florais. 
Agradecimentos. Ao CADCT/SEPLANTEC-BA, PIDCT/CAPES/ UFBA e ao $\mathrm{CNPq}$ pelo apoio financeiro concedido ao projeto de pesquisa; ao Prof. Pe. Jesus Santiago Moure (Universidade Federal do Paraná) pela identificação das espécies de abelhas; ao Prof. Dr. Luciano P. Queiroz (Universidade Estadual de Feira de Santana) pela identificação das espécies vegetais e ao biólogo Ciáxares M. Carvalho pelo auxílio nas atividades de campo.

\section{REFERÊNCIAS}

Ackerman, J. D. 1983a. Specificity and mutual dependency of the orchideuglossine bee interaction. Biological Journal of the Linnean Society 20: 301-304.

ACKerman, J. D. 1983b. Diversity and seasonality of male euglossine bees (Hymenoptera: Apidae) in Central Panama. Ecology 64: 274-283.

ACKerman, J. D. 1989. Geographic and seasonal variation in fragrance choices and preferences of male euglossine bees. Biotropica 20: 301-304.

Agullar, J. B. V. 1990. Contribuição ao conhecimento dos Euglossini (Hymenoptera: Apidae) do estado da Bahia. Dissertação de Mestrado, Instituto de Biociências, Universidade de São Paulo, 96p.

Armbruster, W. S. \& E. E. Berg. 1994. Thermal ecology of male euglossine bees in a tropical wet forest: fragrance foraging in relation to operative temperature. Biotropica 26(1):50-60.

Bezerra, C. P. \& C. F. Martins. 2001. Diversidade de Euglossinae (Hymenoptera, Apidae) em dois fragmentos de mata atlântica localizados na região urbana de João Pessoa, Paraíba, Brasil. Revista Brasileira de Zoologia 18(3): 823-835.

Camargo, J. M. F. \& M. Mazucato. 1984. Inventário da apifauna apícola de Ribeirão Preto, SP. Brasil. Dusenia 14: 55-87.

Campos, L. A. O.; F. A. Silveira; M. L. Oliveira; C. M. V. Abrantes; E. F. Morato \& G. A. R. Melo. 1989. Utilização de armadilhas para captura de machos de Euglossini (Hymenoptera: Apoidea). Revista Brasileira de Zoologia 6(4): 621-626.

Dodson, C. H. 1966. Ethology of some bees of the tribe Euglossini (Hymenoptera: Apidae). Journal of the Kansas Entomological Society 39: 607-629.

Dodson, C. H.; R. L. Dressler; H. G. Hills; R. M. Adams \& N. H. Williams. 1969. Biologically active compounds in orchid fragrances. Science 164(13): 1243-1249.

Dressler, R. L. 1967. Why do euglossine bees visit orchid flowers? Atas do Simpósio sobre a Biota Amazônica 5: 171-180.

Dressler, R. L. 1982. Biology of orchid bees (Euglossini). Annual Review of Ecology and Systematics 13: 373-394.

Heithaus, E. R. 1979. Flower visitation records and resource overlap of bees and wasps in northwest Costa Rica. Brenesia 16: 9-52.

Janzen, D. H.; P. J. Devries; M. L. Higgins \& L. S. Kimsey. 1982. Seasonal and site variation in Costa Rican euglossine bees at chemical baits in lowland deciduous and evergreen forests. Ecology 63: 66-74.

Jesus, B. M. V. 2000. Riqueza e abundância de Euglossini (Hymenoptera, Apidae) em fragmentos de matas do estado de São Paulo. Tese de Doutorado, FFCLRP, Universidade de São Paulo, Ribeirão Preto, 82 p.

Minckley, R. L. \& S. G. Reyes. 1996. Capture of the Orchid bee, Eulaema polychroma (Friese) (Apidae: Euglossini) in Arizona, with notes on northern distribuitions of other mesoamerican bees. Journal of the Kansas Entomological Society 69(1): 102-104.

Montalvo, A. M. \& J. D. Ackerman. 1986. Relative pollinator effectiveness and evolution of floral traits in Spathiphyllum friedrichsthalii (Araceae). American Journal of Botany 73(12): 1665-1676.

Morato, E. F. 1998. Estudos sobre comunidades de abelhas Euglossini. Anais do $3^{\circ}$ Encontro sobre Abelhas de Ribeirão Preto, São Paulo, p.135-143.

Morato, E. F \& L. A. O. Campos. 1992. Abelhas Euglossini (Hymenoptera: Apidae) coletadas na Amazônia central. Revista Brasileira de Entomologia 36(4): 767-771.

Moure, J. S. 1967. A checklist for the known euglossine bees (Hy- menoptera: Apidae). Atas do Simpósio sobre a Biota Amazônica 5: $395-415$

Neves, E. L. \& B. F. Viana. 1997. Inventário da fauna de Euglossinae (Hymenoptera, Apidae) do baixo sul da Bahia, Brasil. Revista Brasileira de Zoologia 14(4): 831-837.

Neves, E. L. \& B. F. Viana. 1999. Comunidade de machos de Euglossinae (Hymenoptera, Apidae) das matas ciliares da margem esquerda do médio Rio São Francisco, Bahia. Anais da Sociedade Entomológica do Brasil 28(2): 201-210.

Oliveira, M. L. 1999. Sazonalidade e horário de atividade de abelhas Euglossinae (Hymenoptera, Apidae) em florestas de terra firme na Amazônia Central. Revista Brasileira de Zoologia 16(1): 83-90.

Oliveira, M. L. \& L. A. O. CAMPos. 1995. Abundância, riqueza e diversidade de abelhas euglossinae (Hymenoptera; Apidae) em florestas contínuas de terra firme na Amazônia central, Brasil. Revista Brasileira de Zoologia 12(3): 547-556.

Oliveira, M. L. \& L. A. O. Campos. 1996. Preferência por estratos florestais e por substâncias odoríferas em abelhas Euglossinae (Hymenoptera, Apidae). Revista Brasileira de Zoologia 13(4): 1075-1085.

Peruquetti, R. C. 2000. Function of fragrances collected by Euglossini males (Hymenoptera: Apidae). Entomologia Generalis 25(1): 33-37.

Peruquetti, R. C.; L. A. O. Campos; C. D. P. Coelho; C. V. M. Abrantes \& L. C. O. LsBoA. 1999. Abelhas Euglossini (Apidae) de áreas de mata atlântica: abundância, riqueza e aspectos biológicos. Revista Brasileira de Zoologia 16(Supl. 2): 101-118.

Pearson, D. L. \& R.L. Dressler. 1985. Two year study of male orchid bee (Hymenoptera, Apidae, Euglossini) attraction to chemical baits in lowland south-eastern Peru. Journal of Tropical Ecology 1: 37 54 .

Raw, A. 1989. The dispersal of euglossine bees between isolated patches of eastern brazilian wet forest (Hymenoptera, Apidae). Revista Brasileira de Entomologia 33(1): 103-107.

Rebêlo J. M. \& C. A. Garófalo. 1991. Diversidade e sazonalidade de machos de Euglossini (Hymenoptera, Apidae) e preferências por iscas odores em um fragmento de floresta no sudeste do Brasil. Revista Brasileira de Biologia 51(4): 787-799.

Rebêlo, J. M. M. \& C. A. Garófalo. 1997. Comunidades de machos de Euglossini (Hymenoptera: Apidae) em matas secundárias do nordeste do estado de São Paulo. Anais da Sociedade Entomológica do Brasil 26(2): 243-255.

RebêLo, J. M. M. \& F. S. Silva. 1999. Distribuição das abelhas euglossini (Hymenoptera: Apidae) no estado do Maranhão, Brasil. Anais da Sociedade Entomológica do Brasil 28(3): 389-401.

RoubiK, D. W. 1989. Ecology and natural history of tropical bees. Cambridge, Cambridge University Press, 514p.

RoubiK, D. W. \& J. D. ACKerman. 1987. Long term ecology of euglossine orchid bees (Apidae: Euglossini) in Panama. Oecologia 73: 321333.

Sakagami, S. F.; S. Laroca \& J. S. Moure. 1967. Wild bee biocenotics in São José dos Pinhais (Pr), South Brazil. Journal of the Faculty of Hokkaido University, VI Zool, 19: 190-250.

Silva, F. S. \& J. M. M. RebêLo. 1999. Euglossine bees (Hymenoptera: Apidae) of Buriticupu, Amazonia of Maranhão, Brazil. Acta Amazônica 29(4): 587-599.

VIANA, B. F. 1999. Biodiversidade da apifauna e flora apícola das dunas litorâneas da APA das lagoas e Dunas de Abaeté, Salvador, Bahia - Composição, fenologia e suas interações. Tese de Doutorado, Instituto de Biociências, Universidade de São Paulo, $171 \mathrm{p}$.

Viana, B. F.; F. O. Silva \& A. M. P. Kleinert. 2001a. Diversidade sazonalidade de abelhas solitárias (Hymenoptera: Apoidea) em dunas litorâneas no nordeste do Brasil. Neotropical Entomology 30(2): 245-251.

Viana, B. F.; E. L. Neves \& F. O. Silva. 2001b. Aspectos da biologia de nidificação de Euplusia mussitans (Fabricius) (Hymenoptera, Apidae, Euglossini). Revista Brasileira de Zoologia 18(4): 1081-1087.

Williams, N. H. 1982. The biology of orchids and euglossine bees, p. 119171. In: J. ARditti (ed.). Orchid biology: reviews and perspec- 
tives, II. Ithaca, Cornell University Press, $610 \mathrm{p}$.

Williams, N. H. \& W. M. Whitten. 1983. Orchid floral fragrances and male euglossine bees: Methods and advances in the last sesquidecade. Biological Bulletin 164: 355-395.

Wittmann, D.; R.RadtKe; M. Hoffmann \& B. Blochtein. 1987. Seasonality and seasonal changes in preferences for chemicals baits of male Eufriesea violacea in Rio Grande do Sul, southern Brazil, p. 730731. In: J. Eder \& H. Rembold (Eds.). Chemistry and biology of social insects. Munique, Verlag J. Peperny, 757p.

Wittmann, D.; M. Hoffmann; E. Scholz. 1988. Southern distributional limits of euglossine bee in Brazil linked to habitats of the Atlantic and Subtropical rain forest (Hymenoptera: Apidae: Euglossini) Entomologia Generalis 14(1): 53-60.

Zimmerman, J. K. \& S. R. MadRinan. 1988. Age structure of male Euglossa imperialis (Hymenoptera, Apidae, Euglossini) at nectar and chemical sources in Panama. Journal of Tropical Ecology 4: 303-306. Zucchi, R.; J. M. F. CAmargo \& S. F. Sakagami. 1969. Biological observations on a neotropical bee, Eulaema nigrita, with a review on the biology of Euglossinae (Hymenoptera: Apoidea): A comparative study. Journal of the Faculty of Hokkaido University, VI Zool, 17: $271-380$ 